\title{
The number line effect reflects top-down control
}

\author{
JELENA RISTIC \\ University of British Columbia, Vancouver, British Columbia, Canada \\ ALISSA WRIGHT \\ University of Toronto, Toronto, Ontario, Canada \\ and \\ ALAN KINGSTONE \\ University of British Columbia, Vancouver, British Columbia, Canada
}

\begin{abstract}
Recent evidence indicates that central directional stimuli, such as eyes and arrows, trigger rapid, reflexive shifts of spatial attention. A study by Fischer, Castel, Dodd, and Pratt (2003) suggested that a similar effect might also apply to central numbers, as if a digit's meaning causes attention to be oriented to its relative position on a left-to-right mental number line. However, unlike central eyes and arrows, the orienting effect for central digits emerges slowly, suggesting that top-down endogenous processes may be mediating this effect. Here, we report a series of three experiments that strongly support this hypothesis. Experiment 1 replicated Fischer et al.'s left-to-right number line effect. Experiment 2 showed that this effect could be completely reversed by merely asking participants to imagine a number line running from right to left. Experiment 3 showed that a left-to-right number line effect could be abolished by presenting targets above and below central fixation, as well as to the left and right of center. Experiment 3 also showed that other mental sets, such as imagining a clock, result in attention's being oriented in accordance with where the central digits are represented on a clock face. Together, these data indicate that the spatial representations and attentional orienting related to the perception of digits are both fragile and flexible and depend critically on the top-down spatial mental sets adopted by individuals.
\end{abstract}

Human attention is normally conceived as a limited capacity process that can be controlled in either an exogenous, reflexive manner or an endogenous, volitional manner (Broadbent, 1971; Posner, 1978). For several decades, the attention-cuing paradigm has provided a simple methodology for engaging and measuring these two forms of orienting (Posner, 1980; Posner, Snyder, \& Davidson, 1980).

Typically, reflexive attention has been triggered by a peripheral flash that does not predict where a visual target will appear. This attentional cue is followed by a target, demanding a manual detection response, appearing either at the cued location or at a noncued location. The usual result is that if the cue-target stimulus onset asynchrony (SOA) is less than $300 \mathrm{msec}$, reaction time (RT) is shorter when a target appears at a cued location versus a noncued

This research was supported by graduate fellowships awarded to J.R. from the Natural Sciences and Engineering Research Council of Canada (NSERC) and the Michael Smith Foundation for Health Research (MSFHR) and by support to A.K. from NSERC, the Hampton and Killam Foundations, MSFHR, and the Human Early Learning Partnership (HELP). The first two authors contributed equally, with first authorship being determined by a coin toss. Correspondence regarding this article should be addressed either to J. Ristic, Department of Psychology, University of British Columbia, 2136 West Mall, Vancouver, BC, V6G 1P5 Canada (e-mail: jelena.ristic@telus.net) or to A. Kingstone, Department of Psychology, University of British Columbia, 2136 West Mall, Vancouver, BC, V6G 1P5 Canada (e-mail: alan.kingstone@ubc.ca). location. Because attended items are processed more efficiently than nonattended items, the facilitation effect at the cued location is taken as evidence that the abrupt onset attracted attention to its location. And because the facilitation effect emerged rapidly in response to a cue that did not predict where the target would appear, the attention effect is considered to be reflexive in nature. For SOAs greater than $300 \mathrm{msec}$, RT becomes longer for targets appearing at the cued location than for those appearing at the noncued locations, reflecting the inhibition-of-return phenomenon. This inhibition effect at the cued location is taken as evidence that attention was withdrawn from the cued location and is inhibited in returning there (Posner \& Cohen, 1984).

The cuing methodology for volitional orienting is similar to the above, with two important exceptions. First, instead of an attentional cue being flashed in the periphery, a central directional cue, usually an arrow, points toward the cued location. Second, the arrow cue predicts where the target is most likely to appear. The usual result when this central predictive cuing methodology is used is that an RT advantage emerges relatively slowly at the cued location and then persists across the long cue-target SOAs, since there is little reason to shift attention away from where the target is most likely to appear (e.g., Jonides, 1981).

Recently, a number of studies have shown that central directional cues do not need to be spatially predictive in 
order to induce a shift in spatial attention to the cued location. Friesen and Kingstone (1998) reported that a simple, spatially nonpredictive central schematic face looking left or right triggers a shift in attention to the gazed-at (cued) location. Because the facilitation effect at the cued location was so rapid, emerging $100 \mathrm{msec}$ or less after the gaze cue had appeared, and because the gaze cue did not predict where the target was likely to appear, Friesen and Kingstone concluded that the observed attentional effect was reflexive in nature (see also Driver et al., 1999; Langton \& Bruce, 1999). Subsequently, other central directional spatially nonpredictive cues, such as finger pointing, head direction (Langton \& Bruce, 2000), and arrow direction (Ristic, Friesen, \& Kingstone, 2002; Tipples, 2002) have been found that produce similar rapid reflexive shifts of attention to the cued target location.

A recent study by Fischer, Castel, Dodd, and Pratt (2003) suggested that similar to these directional cues, numbers contain a spatial component that induces an obligatory reflexive shift in spatial attention to the left or right. In their study, participants were presented, for $300 \mathrm{msec}$, with the number $1,2,8$, or 9 at central fixation. After a cue-target delay ranging from 350 to $800 \mathrm{msec}$ (Experiment 1) or 550 to 1,300 msec (Experiment 2), a target demanding a detection response was presented in the periphery. The results from both of these experiments indicated that although the central number did not predict spatially where a target would appear, targets appearing on the left were detected more quickly if they were preceded by a low number ( 1 or 2 ), and targets appearing on the right were detected more quickly if they were preceded by a high number ( 8 or 9 ). These data suggested to the authors that simply perceiving a digit causes an automatic shift in spatial attention in a manner that is consistent with that digit's relative position on a mental number line running from left (low numbers) to right (high numbers).

Superficially, the data of Fischer et al. (2003) appear to be quite similar to the results found with other central cues. However, one important difference between Fischer et al.'s data and the previous results is that the RT facilitation effect for the central number cues did not emerge until at least $700 \mathrm{msec}$ after a nonpredictive number cue had been presented. This contrasts sharply with the rapid reflexive attention effects obtained with central directional cues such as eyes and arrows (and abrupt peripheral onset cues as well), where the appearance of a cue triggers a shift in attention to the cued location within $100 \mathrm{msec}$. The attentional effect engaged by number cues was far slower to emerge, suggesting that this form of attentional orienting may depend critically on the involvement of top-down strategic mechanisms that subserve volitional attention.

The aim of the present study was to explore this idea in a series of three experiments. Experiment 1 was designed to provide a replication of the results in Fischer et al. (2003). In Experiments 2 and 3, we examined the extent to which the mental number line effect is vulnerable to simple topdown changes in the mental set of the participants - that is, imagining numbers on a line running right to left (Ex- periment 2) or on the face of a clock (Experiment 3). If the effect reported by Fischer et al. is reflexive, it should be relatively resistant to these manipulations; if not, it should be susceptible to these manipulations.

\section{EXPERIMENT 1}

\section{Method}

Participants. Eighteen undergraduates participated. All had normal or corrected vision and were naive as to the purpose of the experiment.

Design. The experiment was modeled on Fischer et al. (2003, Experiment 1). There were four central digit cues (1, 2, 8, and 9), two target locations (left and right), and six cue-target SOAs (350, $400,500,600,700$, and $800 \mathrm{msec}$ ). Cues, locations, and SOAs were selected randomly. The experiment consisted of six experimental blocks of 80 trials.

Procedure. The participants were seated and centered with respect to a 14-in. monitor and a keyboard connected to a $6100 / 66$ Macintosh computer. Figure 1 illustrates the stimuli (white on black) and a sequence of events used in Experiments 1-3. All the trials began with the presentation of a $0.1^{\circ}$ central dot flanked by two boxes, subtending $1^{\circ}$ and positioned $5^{\circ}$ to the left and right of center. After $500 \mathrm{msec}$, the fixation point was replaced by one of the digits, which was presented for $300 \mathrm{msec}$ and then replaced by the fixation point. On $80 \%$ of the trials, a $0.7^{\circ}$ white circle serving as the target appeared in one of the boxes. The display was terminated, signaling the end of a trial, with a response execution or after the target had been displayed for $1,000 \mathrm{msec}$, whichever came first. On the remaining $20 \%$ of the trials, a target was not presented (catch trials), and the display was extinguished $1,800 \mathrm{msec}$ after cue onset. The intertrial interval was $1,500 \mathrm{msec}$. The participants were verbally instructed to fixate the center of the screen throughout a trial and to press the space bar as quickly as possible when a target was detected. If no target appeared, they were to wait for the next trial. The participants were instructed that the digits did not predict target location. Eye movements were not monitored, since participants avoid movements in detection tasks of this type (Fischer et al., 2003; Kingstone \& Pratt, 1999).

\section{Results}

For all the experiments, response errors (RTs $<$ $100 \mathrm{msec}$, target misses, and false alarms) were rare (mean $<1.1 \%$ /participant). Hence, participants with error rates exceeding 5\% were replaced ( 4 of 76 ). Mean correct RTs for Experiment 1 are illustrated in Figure 2A.

An ANOVA was conducted on mean correct RTs, with SOA (350-800 msec), target location (left or right), and digit cue (low or high) as within-subjects variables. The main effect of SOA was significant $[F(5,85)=6.04, p<$ $.0001]$, reflecting a classic foreperiod effect (Mowrer, 1940). Replicating Fischer et al. (2003), a significant target location $\times$ digit cue interaction $[F(1,17)=5.48, p<.05]$ was recorded, with targets detected most quickly overall on the left when the digit cue was low (1 or 2) and most quickly on the right when the digit cue was high ( 8 or 9 ). No other interactions were significant (all $p \mathrm{~s}>.1$ ).

\section{Discussion}

The results replicate those in Fischer et al. (2003). Nonpredictive central digits induce attention to be shifted to the left or right, so that when the digit is low in magnitude, attention is shifted to the left, and when the digit is high 
Experiments 1 and 2

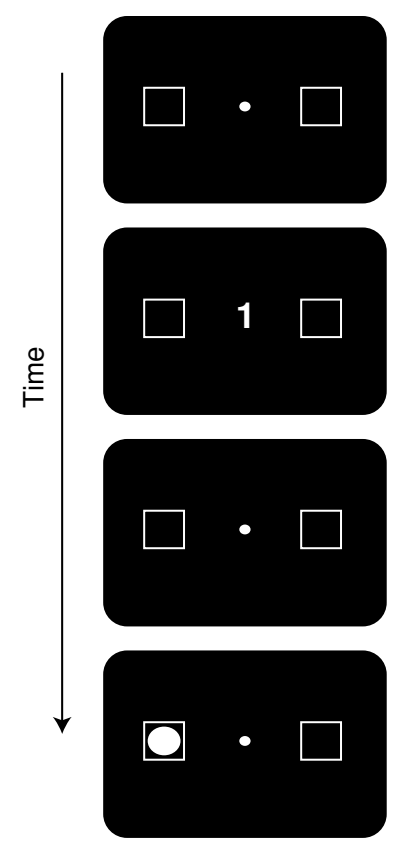

Experiment 3

Fixation Display $500 \mathrm{msec}$

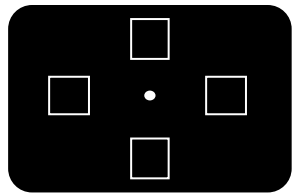

Cue

$300 \mathrm{msec}$

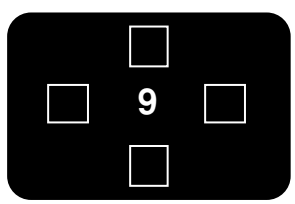

Time Delay

$50,100,200$,

300,400 , or $500 \mathrm{msec}$

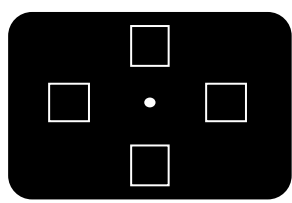

Target

1,000 msec or

Until Response

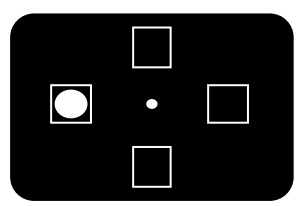

Figure 1. Illustration of the stimuli and a sample sequence of events. At the start of each trial, a fixation point appeared on the screen for 495 msec. Then a number cue $(1,2,8$, or 9 in Experiments 1 and 2 or 1, 3, 6, or 12 in Experiment 3) appeared. After $300 \mathrm{msec}$, the central cue was replaced by the fixation point. The target, demanding a detection response, appeared after a variable cue-target stimulus onset asynchrony of $350,400,500,600,700$, or $800 \mathrm{msec}$. In Experiments 1 and 2 (shown in the left panel), the target could have appeared either in the left or the right placeholder. In Experiment 3 (shown in the right panel), there were four possible target locations (left, right, up, or down). In all the experiments, the target remained on the screen until a response was made or $1,000 \mathrm{msec}$ had elapsed, whichever came first. The intertrial interval was 1,500 msec. Note: The stimuli are not drawn to scale.

in magnitude, attention is shifted to the right. Although the participants were told explicitly that the numbers did not predict where a target would appear and although they were not instructed to imagine a number line running from left to right, their attention was allocated to the left and right as if the perception of the digit caused attention to be directed to that digit's relative position on a mental number line.

\section{EXPERIMENT 2}

In Experiment 2, we examined whether imagining a number line running from right to left would reverse the left-to-right mental number line effect observed in Experiment 1 . Such a reversal would indicate that this mental number line effect is vulnerable to a simple top-down manipulation. As such, this outcome would suggest that the left-to-right mental number line effect is not reflexive in nature. On the other hand, a finding that the mental number line effect of Experiment 1 persisted in Experiment 2 would provide compelling support for Fischer et al.'s (2003) proposal that the left-to-right number line effect is, in fact, automatic.

\section{Method}

Eighteen new naive undergraduates participated. All the experimental parameters were identical to those in Experiment 1. The participants were verbally instructed to imagine a number line running from right to left, with low numbers positioned on the right and high numbers positioned on the left. Again, they were asked to press the space bar as quickly as possible when a target was detected. As before, it was emphasized that the digits did not predict target location.

\section{Results}

The data were analyzed as before. The results revealed a main effect of SOA $[F(5,85)=11.11, p<.0001]$, indicating a foreperiod effect. Most important, there was again a significant target position $\times$ digit interaction $[F(1,17)=$ $5.29, p<.05]$, with targets detected most quickly overall on the left when preceded by a high digit (8 or 9) and most quickly on the right when preceded by a low digit (1 or 2). As in Experiment 1, no other effects were significant (all $p \mathrm{~s}>.1$ ). The key and only meaningful difference between Experiment 1 and Experiment 2 was that the number line effect in Experiment 2 was in the opposite direction from the number line effect in Experiment 1. Consistent with this conclusion, a between-groups ANOVA (Experiment 1 

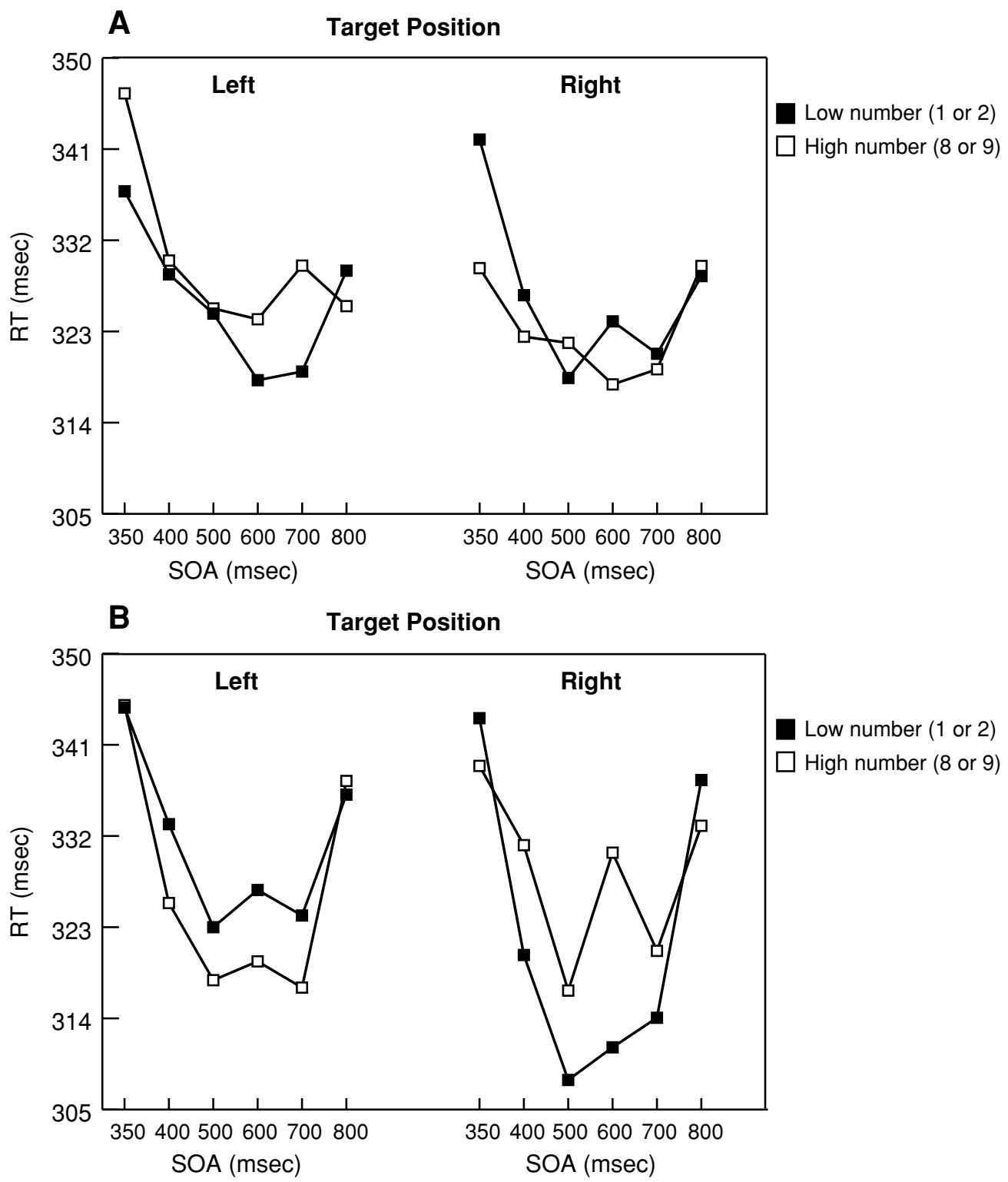

Figure 2. Experiment 1 and Experiment 2 reaction time (RT) results. Figure 2A illustrates Experiment 1 mean correct RTs for targets appearing to the left and right of center as a function of digit cue magnitude (low/high) and stimulus onset asynchrony (SOA). Figure 2B illustrates Experiment $\mathbf{2}$ mean correct RTs for targets appearing to the left and right of center as a function of digit cue magnitude (low/high) and SOA.

vs. Experiment 2) yielded a significant experiment $\times$ target position $\times$ digit cue interaction $[F(1,34)=10.43, p<$ $.005]$. No other effects were significant (all $p \mathrm{~s}>.09$ ), with the exception of a main effect of SOA $[F(5,170)=$ $16.23, p<.0001]$.

\section{Discussion}

Fischer et al. (2003) proposed that their results, which we replicated in Experiment 1, suggested that "a spatially oriented 'mental number line' [running from left to right] is automatically activated as part of a number's meaning whenever we look at numbers" (p. 555). Our findings in Experi- ment 2 argue strongly against this proposal. Merely asking the participants to imagine a mental number line running from right to left was sufficient to generate a number line effect that was in the direction opposite to the one observed in Experiment 1. This suggests that the mental number line effect is not reflexive but, rather, reflects top-down spatial representations imposed on a situation by participants.

\section{EXPERIMENT 3}

Experiment 1 showed that when participants are not instructed to imagine a mental number line, attention is 
shifted to the left when they are perceiving low digits and attention is shifted to the right when they are perceiving high digits. Experiment 2 demonstrated that this left-to-right number line effect can be reversed by asking participants to imagine a number line running right to left. Do these two experiments together suggest that numbers always activate a left-right number line spatial representation when there is no explicit instruction otherwise? We have reason to think that this is not the case (e.g., Bächtold, Baumüller, \& Brugger, 1998; Ristic \& Kingstone, 2006). Ristic and Kingstone (2006) found that when participants are presented with number cues, such as 1 and 9 , and possible target locations are positioned above and below center, as well as to the left and right of center, the number 1 does not produce a shift in attention to the left and the number 9 does not produce a shift in attention to the right. If simply adding a top target location and a bottom target location to the current display is sufficient to abolish the left-to-right number line effect (possibly because left-right are no longer afforded a special status, relative to top-bottom), this would indicate that numbers need not always activate a left-right number line spatial representation.

One aim of Experiment 3 was to test this possibility by adding a top target location and a bottom target location to the display. Another aim of Experiment 3 was to test the conclusion reached at the end of Experiment 2, that a simple top-down mental set can have a significant impact on how attention is oriented in response to central digits. To do this, we moved beyond the idea of a mental number line and asked a second group of participants to imagine that the four target locations in Experiment 3 were distinct points on a clock. We predicted that such a mental set would result in attention's being shifted in accordance with where a digit cue's form is represented on a clockthat is, to the right when the cue is a 3 , to the left when the cue is a 9 (note that this is opposite to the putative default mental number line), to the bottom when the cue is a 6 , and to the top when the cue is a 12 .

\section{Method \\ Thirty-six new and naive undergraduates were divided equally be- tween two groups of 18 participants: a no-instructions group and an instructions group. All aspects remained as before, with the follow- ing exceptions. The central number cues were 3, 6, 9, and 12. Two additional locations, matched in size and distance to those on the left and right, were positioned above and below center (see Figure 1). Finally, the instructions given to the no-instructions group paralleled that given to the participants in Experiment 1. The information given to the instructions group paralleled that given to the participants in Experiment 2, except that the participants were asked to imagine a clock face, instead of a number line, when the cue appeared on the screen. As before, it was emphasized that the digits did not predict a target's location.}

\section{Results}

The analyses were the same as before, although now it was a 6 (SOA) $\times 4$ (target location) $\times 4$ (digit cue) ANOVA.

For the no-instructions group, there was a significant SOA main effect $[F(5,85)=3.65, p<.01]$, reflecting a foreperiod effect. Figure 3 shows that there was also an effect of target position $[F(3,51)=7.52, p<.001]$, with overall RT shortest for targets on the right and longest overall for targets presented on the bottom, with the other locations falling in between. Different digits had no reliable effect on RT and did not interact with target location (all $F_{\mathbf{S}}<1$ ). No other effects were significant (all $F_{\mathbf{S}}<$ 1.2 , all $p \mathrm{~s}>.2$ ).

For the instructions group, there was a significant SOA foreperiod effect $[F(5,85)=13.04, p<.0001]$. Most important, the target location $\times$ digit cue interaction $[F(9,153)=4.59, p<.0001]$ was significant, reflecting the fact that the participants responded most quickly to a target that appeared at the location on a clock face that was congruent with the digit cue; that is, RT was shortest for a target at the top when the digit cue was a 12, a target on the right when the digit cue was a 3 , a target on the bottom when the digit cue was a 6 , and a target on the left when the digit cue was a 9. As is illustrated in Figure 3, this location-cue congruency effect interacted with SOA $[F(45,765)=1.54, p<.02]$, with the congruency effect increasing in magnitude as the SOA lengthened toward 700 msec. (Note that Fischer et al., 2003, reported a similar time course for their mental number line congruency effect.) No other effects were significant (all $F \mathrm{~s}<1.6$, all $p \mathrm{~s}>.2)$.

\section{Discussion}

The results of Experiment 3 are unequivocal. With four possible target locations - top, right, bottom, and leftand no explicit instructions regarding a particular mental set, the digits failed to have any reliable effect on the allocation of attention; for example, there was no mental number line effect. Importantly, when the participants were asked to imagine the digits on a clock face, attention was allocated to a spatial position congruent with a digit's position on a clock's face.

These data make at least two important points. One is that the "default" left-to-right number line effect, reported by Fischer et al. (2003) and observed in Experiment 1, is not reflexive enough to survive simply balancing the left-right target display with top-bottom target locations. Second, a mental number line effect can be readily subsumed by a very different mental set, such as imagining a clock (see also Bächtold et al., 1998).

\section{GENERAL DISCUSSION}

Recently, investigations have revealed that central directional stimuli, such as arrows and gaze, trigger reflexive shifts of attention. Two key criteria indicative of reflexive orienting have been met by these stimulus cues: (1) They reliably trigger shifts in spatial attention even though participants know that they do not predict a target's location, and (2) they reliably trigger a rapid shift in spatial attention, in less than 100 or $200 \mathrm{msec}$ (e.g., Friesen $\&$ Kingstone, 1998; Tipples, 2002). An interesting finding reported by Fischer et al. (2003) suggested that numbers may also trigger a reflexive shift in spatial attention by automatically activating a left-to-right mental number line 


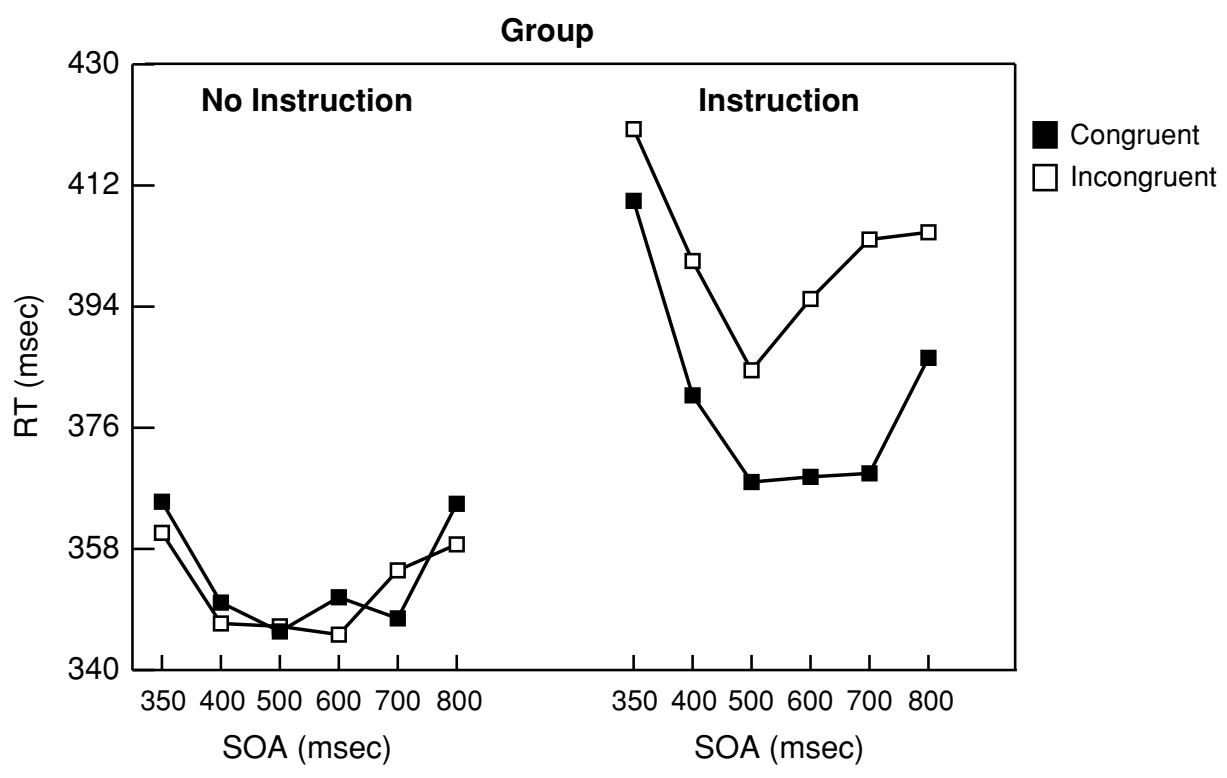

Figure 3. Experiment 3 results. Figure 3 shows mean correct reaction times (RTs) as a function of stimulus onset asynchrony (SOA) and digit-cue-target-location congruency relative to a clock face (e.g., a target at the top was congruent when the cue was a 12, and a target on the bottom was congruent when the cue was a 6). The no-instructions group, illustrated on the left, were not told to imagine a clock's face, and the instructions group, illustrated on the right, were instructed to imagine a clock's face.

"as part of number's meaning whenever we look at numbers" (p. 555). Importantly, although Fischer et al.'s result seemed to satisfy the first criterion for reflexive orienting, it did not satisfy the second criterion, since the mental number line effect emerged slowly, about $700 \mathrm{msec}$ after the digit cue first appeared. This slow time course of activation suggests the involvement of volitional, top-down attentional orienting. The present study pursued this issue, testing whether the mental number line effect is reflexive and insensitive to top-down control, like gaze direction (Driver et al., 1999; Friesen, Ristic, \& Kingstone, 2004).

The data from the present investigation were unequivocal on this issue. The effect exerted by digits is not reflexive but, rather, is extraordinarily susceptible to top-down changes in mental set. After Fischer et al.'s (2003) result had been replicated in Experiment 1, with low numbers biasing attention to the left and high numbers biasing attention to the right, Experiment 2 showed that this leftto-right effect could be reversed simply by asking the participants to imagine a number line running from right to left. Experiment 3 showed that a left-to-right number line effect could also be abolished by presenting targets above and below central fixation, in addition to presenting them to the left and right of center. Finally, the results of Experiment 3 demonstrated that other mental sets, such as imagining a clock, result in attention's being oriented in accordance with where the digits are depicted on a clock's face (see also Bächtold et al., 1998).

In sum, although nondirectional central digit cues, such as numbers, can produce a shift in attention that is suggestive of the reflexive attention effect typically obtained with directional central cues, the evidence indicates that attentional orienting to digit cues is critically mediated by strategic, top-down factors. That is, the mere perception of a digit does not appear to activate a spatially represented number line, nor does the perception of a digit produce any obligatory shifts in spatial attention (see also Galfano, Rusconi, \& Umiltà, 2006, for a similar conclusion). Instead, the spatial representation triggered by the perception of a digit appears to depend crucially on the spatial mental set that is currently being adopted by an individual.

In conclusion, it appears that there are a range of stimuli that can produce "reflexive" shifts in spatial attention. Some, such as abrupt peripheral onsets and gaze direction, trigger reflexive shifts of attention that are difficult to override (Friesen et al., 2004; Ristic \& Kingstone, 2005; Yantis \& Jonides, 1990). Others, such as arrows, produce rapid shifts of attention even when they are known to be spatially nonpredictive, but they can be overridden by topdown control more easily than comparable stimuli-for example, gaze direction (Friesen et al., 2004). At the other end of the spectrum are stimuli, such as numbers, that can produce shifts of attention when they are spatially nonpredictive, but this orienting effect is both slow to emerge and extremely sensitive to changes in the top-down mental set adopted by a participant.

\section{REFERENCES}

B̈̈Chtold, D., Baumüller, M., \& Brugger, P. (1998). Stimulusresponse compatibility in representational space. Neuropsychologia, 36, 731-735. 
Broadbent, D. E. (1971). Decision and stress. London: Academic Press. Driver, J., Davis, G., Ricciardelli, P., Kidd, P., Maxwell, E., \& BARON-CoHEN, S. (1999). Gaze perception triggers visuospatial orienting. Visual Cognition, 6, 509-540.

Fischer, M. H., Castel, A. D., Dodd, M. D., \& Pratt, J. (2003). Perceiving numbers causes spatial shifts of attention. Nature Neuroscience, 6, 555-556.

Friesen, C. K., \& Kingstone, A. (1998). The eyes have it! Reflexive orienting is triggered by nonpredictive gaze. Psychonomic Bulletin \& Review, 5, 490-495.

Friesen, C. K., Ristic, J., \& Kingstone, A. (2004). Attentional effects of counterpredictive gaze and arrow cues. Journal of Experimental Psychology: Human Perception \& Performance, 30, 319-329.

Galfano, G., Rusconi, E., \& Umiltà, C. (2006). Number magnitude orients attention, but not against one's will. Psychonomic Bulletin \& Review, 13, 869-874.

JoNIDES, J. (1981). Voluntary versus automatic control over the mind's eye's movement. In J. [B.] Long \& A. [D.] Baddeley (Eds.), Attention and performance $I X$ (pp. 187-203). Hillsdale, NJ: Erlbaum.

Kingstone, A., \& PratT, J. (1999). Inhibition of return is composed of attentional and oculomotor processes. Perception \& Psychophysics, 61, 1046-1054.

LANGTON, S. R. H., \& BRUCE, V. (1999). Reflexive social orienting in response to the social attention of others. Visual Cognition, 6, 541-567.

Langton, S. R. H., \& BRUCE, V. (2000). You must see the point: Automatic processing of cues to the direction of social attention. Journal of Experimental Psychology: Human Perception \& Performance, 26, 747-757.

Mowrer, O. H. (1940). Preparatory set (expectancy)—some methods of measurement. Psychological Review Monographs, 52 (2, Whole No. 233).

PosNer, M. I. (1978). Chronometric explorations of the mind. Hillsdale, NJ: Erlbaum.

Posner, M. I. (1980). Orienting of attention. Quarterly Journal of Experimental Psychology, 32, 3-25.

Posner, M. I., \& CoHEN, Y. (1984). Components of visual orienting. In H. Bouma \& D. G. Bouwhuis (Eds.), Attention and performance X: Control of language processes (pp. 531-556). Hillsdale, NJ: Erlbaum.

Posner, M. I., SNyder, C. R. R, \& Davidson, B. J. (1980). Attention and the detection of signals. Journal of Experimental Psychology: General, 109, 160-174.

Ristic, J., Friesen, C. K., \& Kingstone, A. (2002). Are eyes special? It depends on how you look at it. Psychonomic Bulletin \& Review, 9 , 507-513.

Ristic, J., \& KingSTONE A. (2005). Taking control of reflexive social attention. Cognition, 94, B55-B65.

Ristic, J., \& Kingstone, A. (2006). Attention to arrows: Pointing to a new direction. Quarterly Journal of Experimental Psychology, 59, 1921-1930.

Tipples, J. (2002). Eye gaze is not unique: Automatic orienting in response to uninformative arrows. Psychonomic Bulletin \& Review, 9, 314-318.

YAntis, S., \& Jonides, J. (1990). Abrupt visual onsets and selective attention: Voluntary versus automatic allocation. Journal of Experimental Psychology: Human Perception \& Performance, 16, 121-134.

(Manuscript received July 21, 2005;

revision accepted for publication December 19, 2005.) 